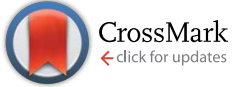

Cite this: Soft Matter, 2015, 11, 1800

Received 19th September 2014

Accepted 9th January 2015

DOI: $10.1039 / \mathrm{c} 4 \mathrm{sm} 02098 \mathrm{~g}$

www.rsc.org/softmatter

\section{Jammed elastic shells - a 3D experimental soft frictionless granular system $\uparrow$}

\author{
Jissy Jose,,$^{\star a}$ Gerhard A. Blab, Alfons van Blaaderen ${ }^{a}$ and Arnout Imhof ${ }^{\star a}$
}

We present a new experimental system of monodisperse, soft, frictionless, fluorescent labeled elastic shells for the characterization of structure, universal scaling laws and force networks in 3D jammed matter. The elastic shells in a jammed packing are deformed in such a way that at each contact one of the shells buckles with a dimple and the other remain spherical, closely resembling overlapping spheres. Using confocal microscopy, we obtained 3D stacks of images of shells at different volume fractions which were subsequently processed in ImageJ software to find their coordinates. The determination of 3D coordinates involved three steps: locating the edges of shells in all 2D slices, analyzing their shape and subsequently finding their 2D coordinates, and finally determining their 3D centers by grouping the corresponding 2D coordinates. From this analysis routine we obtained particle coordinates with subpixel accuracy. In a contact pair we also identified the shell that underwent buckling forming a dimple by analyzing the intensity profile of a line that connects the centers of particle pairs. The amorphous structure of the packing was analyzed as a function of distance to the jamming threshold by investigating the radial distribution function, bond order parameters, contact numbers and the number of dimples per particle (buckling number), which is a unique property of this system. We find that the power law scaling of the contact number with excess volume fraction deviated from theoretical and computer simulation predictions. In addition, the buckling number also showed a similar scaling as that of the contact number with distance to the jamming transition.

\section{Introduction}

The term jamming refers to the phenomenon where constituent particles, whether they be molecular, colloidal or granular, in their disordered state are arrested in a small region of phase space with a long stress relaxation time that exceeds the experimental time scale. ${ }^{1,2}$ Above the jamming point the collection of particles behaves like an elastic solid and below this point the system behave like a liquid. This liquid to solid transition (jamming transition) occurs in a wide variety of soft materials such as colloids, granular materials, emulsions and bubbles. The most simple and widely studied model system that illustrates the micro mechanical and geometric properties of

${ }^{a}$ Soft Condensed Matter, Debye Institute for NanoMaterials Science, Utrecht University, Princetonplein 1, 3584 CC, Utrecht, The Netherlands. E-mail:j.jose@uu.nl; A.Imhof@ uu.nl

${ }^{b}$ Molecular Biophysics, Debye Institute for NanoMaterials Science, Utrecht University, Princetonplein 1, 3584 CC, Utrecht, The Netherlands

$\dagger$ Electronic supplementary information (ESI) available: Atomic force microscopy (AFM) measurement on elastic shell to determine the shell thickness. Plots of radius of smallest enclosing circles as a function of position along the axial direction for a typical ambiguous particle and particles with several dimples. Histograms of fractional part of position coordinates to check for pixel biasing. See DOI: $10.1039 / \mathrm{c} 4 \mathrm{sm} 02098 \mathrm{~g}$

\$ Present address: Physics and Physical Chemistry of Foods, Wageningen UR, Bornse Weilanden 9, 6708 WG, Wageningen, The Netherlands jammed matter is a system consisting of frictionless soft spheres at zero temperature and zero shear, interacting with finite-range repulsive potentials. In such a system the onset of jamming occurs when the spheres just start to touch each other, but still at zero pressure, at a critical volume fraction $\varphi_{\mathrm{c}}$ that was found to coincide with random close packing fraction RCP $\approx$ $0.64 .^{3,4}$ Above $\varphi_{\mathrm{c}}$, the particles start to overlap and exert forces on each other. Hence the pressure becomes non-zero and the system starts to become rigid. The emergence of rigidity is attributed to a force network, which forms the skeleton of jammed matter., ${ }^{1,2}$ Unlike crystalline solids, mechanical and geometric properties of a jammed solid were found to exhibit anomalous behavior with excess volume fraction $\left(\varphi-\varphi_{\mathrm{c}}\right)$. For example, in simulations on frictionless spheres the contact number $Z$ is found to scale as $\left(\varphi-\varphi_{\mathrm{c}}\right)^{\beta}$ above $\varphi_{\mathrm{c}}$, where $\beta \sim 0.5$, regardless of dimension and interaction potential. ${ }^{3-8}$ While there are many theoretical and simulation works that studied the scaling laws and force network in jammed matter; there are only a few experimental systems where these features are quantitatively analyzed, either in 2D or even fewer in 3D.

One of the experimental scaling laws of contact number and pressure was identified and compared with simulation predictions in a $2 \mathrm{D}$ granular system of photoelastic disks. ${ }^{9}$ This system of disks allowed accurate determination of particle contacts and also contact forces in the bulk, ${ }^{10}$ which was earlier limited to 
only the boundary of the system..$^{\mathbf{2 , 1 1 - 1 4}}$ The deformation per disk was less than $1 \%$ of their average diameter in the compressed state, ${ }^{9}$ which prevented the system from reaching higher volume fractions, far beyond the jamming point. Soft deformable particles are interesting in this aspect, as these particles can be packed to reach in principle a volume fraction of unity. Compressed emulsions and foams are the two most widely studied experimental soft frictionless systems to date, of which studies on compressed emulsions were performed in $3 \mathrm{D} .{ }^{15-24}$ These latter studies have produced a detailed characterization of jammed packings of spherical particles. There are not many other soft particles available today to study the nature of jammed matter experimentally. Furthermore, it would be interesting to have a system with a solid surface like granular particles but soft enough to undergo large deformations like emulsions and foams. Charged spherical particles with a solid elastic polymer shell have these properties. Elasticity of the shell allows these particles to act as sensors of local stress. Besides, by tuning the ratio of shell thickness to particle radius, the mechanical behavior of the shells can be varied from soft and floppy to stiff and hard. ${ }^{25,26}$ Additionally, the surface of the shell could be tuned from smooth to rough by surface coatings to adjust the friction between particles. ${ }^{27,28}$ The shells can even be labeled with a fluorescent dye and can be made transparent by index matching such that the dense packing could be imaged in 3D using confocal microscopy. ${ }^{29-34}$ Due to all these features particles with a shell topology, next to other soft particles, foams and emulsion droplets, are an interesting new subclass of soft granular systems for jammed matter research.

Confocal microscopy has been extensively used in colloid science, in particular to obtain quantitative information on a single particle level..$^{35-40}$ The key feature of this technique is the use of a pinhole aperture that is confocal with the focal plane. The pinhole rejects most of the out-of-focus light arriving from the sample, producing a sharper image that represents a thin cross-section of the sample. $3 \mathrm{D}$ reconstruction of the sample is achieved by assembling a series of thin optical slices taken along the axial direction. Confocal microscopy coupled with image analysis techniques has provided a wealth of information on the structure of colloidal crystals, glasses and gels and their behavior under external fields. ${ }^{35-40}$ Many of such quantitative studies are performed on spherical colloids that are homogeneously labeled with a fluorescent dye. The coordinates of these particles can be measured with sub-pixel accuracy ${ }^{35-39}$ using image processing algorithms similar to those developed by Crocker and Grier. ${ }^{41}$ The algorithm works by searching for the local brightness maxima in the image within a characteristic length scale and determine the candidate particle locations, i.e. the nearest pixel to the true location as the starting value. Subsequently, the position is refined to sub-pixel accuracy by taking the first moment of the intensity distribution within a window around each candidate particle location. This algorithm was also extended to locate positions of anisotropic colloids like ellipsoids made by stretching spherical colloids, ${ }^{42}$ rod-like colloidal particles, ${ }^{\mathbf{4 3 , 4 4}}$ and colloidal clusters built up from spherically symmetric blocks, ${ }^{\mathbf{4 5 - 4 8}}$ that are in almost all cases uniformly labeled. As far as we know there is no straightforward routine that can identify particles with a coreshell morphology where the shell is the only part that is fluorescent labeled.

In this paper we present a 3D experimental system consisting of micron size spherical particles with a semipermeable elastic shell of thickness several tens of nanometers for jammed matter research. Here we focus on methods to identify particle coordinates and on the analysis of their amorphous structure as a function of distance to the jamming transition. The elastic shells are prepared by coating polydimethylsiloxane (PDMS) oil droplets with a tetraethoxysilane-crosslinked-polydimethylsiloxane (TC-PDMS) shell and replacing the liquid interior with an index-matching mixture after removing the droplets by dissolution in ethanol. ${ }^{49}$ Due to their reversible elastic response these TC-PDMS shells or capsules (filled with PDMS), have been reported to efficiently encapsulate and release apolar liquids. ${ }^{50}$ Shells can be considered frictionless because the shell surface was found to be smooth, as observed by scanning electron microscopy and in addition there is a layer of liquid between every two contacting shell surfaces containing the electrical double layer. Hence they interact through charge repulsions at short distances and can be re-suspended to form a stable colloid. Using laser scanning confocal microscopy in fluorescence mode we could image deep in the sample, well over 50 $\mu \mathrm{m}$, and acquire 3D stacks of images of index matched shells. Static dense packings of shells were prepared by centrifugation. The system was jammed such that all the particles remained static and stable on our experimental time scale, except for a few percent of rattlers that underwent Brownian motion in the void space between contacts, at low densities close to random close packing.

At high packing fractions the shells underwent large deformation and formed dimples at the contact areas. In other words, the volume of the shells was not conserved in a jammed state. This is different from the case of emulsions and foams, jammed packings of which have also been studied. ${ }^{8,15-17,19}$ The formation of dimples on the shells resulted in asymmetrical shapes. This asymmetry both in shape of these deformable shells and in intensity distribution precluded a straightforward use of available particle finding and tracking algorithms. Therefore, we developed new routines to analyze these particles in 3D. This was made possible by the fact that the deformed particle's shape can be well described by a spherical envelope containing one or more spherical dimples, like overlapping spheres. The analysis routine is based on processing of the $3 \mathrm{D}$ stack of images, where each particle is distributed in a few slices, using ImageJ algorithms. ${ }^{51-53}$ The algorithms take into account the edge and shape of the individual shells in 2D slices to reconstruct the particle in 3D. In addition to finding the particle coordinates, we identified the shell that has been dimpled in each contact pair by measuring the distance of the fluorescence intensity maxima along the line that connects the center of contact pairs from the center of each particle. With knowledge of the particle coordinates, we investigated the amorphous structure of packings of our shells, especially the radial distribution functions $g(r)$ and the average contact number per particle, $Z$ as a function of distance to the jamming 
transition. A check for any local order in these packings was also performed by calculating local bond order parameters. ${ }^{54-56}$ Finally, we studied the distribution of number of dimples per particle (buckling number), which is a unique property of this system, and analyzed the randomness of the process of dimpling one way or the other. A detailed study on measuring/ analysing the local stress between the shells and contact force distribution as a function of distance to the jamming transition will be reported in a follow up paper. ${ }^{57}$

\section{Experimental}

\subsection{Sample preparation}

Monodisperse elastic shells were prepared in three steps: first a polydimethylsiloxane (PDMS) oil-in-water emulsion (total volume $750 \mathrm{~mL}$ ) was made by mixing $6.8 \% \mathrm{v} / \mathrm{v}$ of dimethyldiethoxysilane (DMDES, Sigma Aldrich, 97\%) monomer and 22.7\% $\mathrm{v} / \mathrm{v}$ ammonia (25 wt\% $\mathrm{NH}_{3}$, Sigma Aldrich) using a Turrax homogenizer. ${ }^{49}$ In the second step a tetraethoxysilane-crosslinked-polydimethylsiloxane shell (TC-PDMS) was coated around the droplets by adding $0.33 \mathrm{mM}$ tetraethoxysilane (TES, Sigma Aldrich, 98.0\%) and 0.43 mM DMDES simultaneously after diluting the emulsion $(550 \mathrm{~mL})$ with an aqueous solution (total volume $1725 \mathrm{~mL}$ ) of $2.3 \mathrm{wt} \%$ polyvinylpyrrolidone (PVP, $M_{\mathrm{w}}=58000 \mathrm{~g} \mathrm{~mol}^{-1}$, Sigma Aldrich). The shell was fluorescent labelled by adding a solution of $(0.33 \mathrm{~mL})$ rhodamine B-isothiocyanate (RITC, Sigma-Aldrich) dye to $(1725 \mathrm{~mL})$ reaction medium during shell growth. Before incorporation of the RITC dye into the shells, the silane coupling agent 3-aminopropyltriethoxysilane (APS, Sigma Aldrich) and dye RITC were covalently coupled by mixing overnight $6.5 \mathrm{mg}$ RITC powder and 40 $\mu \mathrm{L}$ coupling agent 3-aminopropyl-triethoxysilane (APS, Sigma Aldrich) in $1 \mathrm{~mL}$ ethanol (100\%, technical grade). Finally, after 2 days of coating the oil filled shells were transferred to anhydrous ethanol to dissolve the PDMS core.

We obtained amorphous packings of deformable shells at different volume fractions by centrifugation. For this purpose we prepared two capillaries (capillaries 1 and 2) from the same batch of particles and a third from another batch (capillary 3 , described below). Capillaries 1 and 2 contained particles of total radius $R_{\mathrm{t}}=2.88 \pm 0.03 \mu \mathrm{m}$ with polydispersity $\sim 3 \%$ and shell thickness $d=56 \pm 2 \mathrm{~nm}$. This corresponds to a shell thickness to particle radius of $d / R_{\mathrm{t}}=0.02$. The radius of the particle was measured by static light scattering (SLS) and the shell thickness was obtained from atomic force microscopy (AFM) (ESI Fig. S1 $\dagger$ ). For confocal measurements the shells were dispersed in an index matched solvent of $52.6 \% \mathrm{v} / \mathrm{v}$ dimethyl sulfoxide (DMSO) and $47.4 \% \mathrm{v} / \mathrm{v}$ ethanol. This mixture had a refractive index of $n_{\mathrm{D}}^{20}=1.42$. A few drops of concentrated suspension of shells were put in two capillaries of dimensions $0.1 \times 2 \times 50$ $\mathrm{mm}$ (capillary 1), and $0.1 \times 1 \times 50 \mathrm{~mm}$, (capillary 2) (depth $\times$ width $\times$ length, VitroCom) both with a wall thickness $0.1 \mathrm{~mm}$. A few millimeters on one side of both the capillaries was filled with candle wax. Both ends of the capillaries were then closed with UV glue (Norland, no. 68) and the glue was cured under a UV lamp for $40 \mathrm{~min}$. The candle wax acted as a pedestal that lifted the whole sediment above the drop of glue. If wax is not

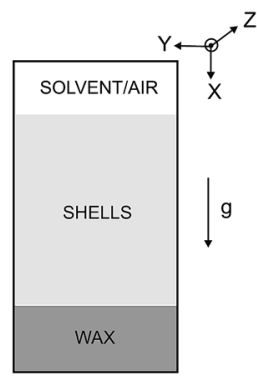

Fig. 1 Schematic of the capillary after centrifugation. The shells sediment in the direction of the centrifugal field (as shown by the arrow next to $g$ ). The leakage of solvent through the wax plug resulted in either a small amount of surplus solvent above the sediment or an air bubble. During imaging the capillary was placed in an upright position with gravity pointing down (as shown by the arrow next to $g$ ).

used as a pedestal, then unfortunately when shells sediment under $g$ force, the high density regions will be hidden under the glue which makes the imaging of dense packing of shells difficult. A schematic of a typical capillary, after centrifugation is shown in Fig. 1.

Capillary 1 was centrifuged at $2000 \mathrm{~g}$ for $10 \mathrm{~min}$ with the capillary length along the direction of gravity. This created a sediment of height $7 \mathrm{~mm}$ with higher densities of shells closer to the wax interface. The density decreased with increasing height in the sediment, moving away from the wax interface. In addition to serving as a support for the sediment, we found that the wax tended to take up a small amount of solvent resulting in some solvent leakage from the capillary during centrifugation. This solvent leakage further enhanced the density of the shells and their subsequent deformation near the vicinity of waxsediment interface. The capillary still contained a surplus of solvent on top of the sediment after centrifugation.

Capillary 2 was centrifuged twice: at $2000 \mathrm{~g}$ for $25 \mathrm{~min}$ and $1000 \mathrm{~g}$ for $15 \mathrm{~min}$, because after the first centrifugation there was no leakage of solvent and hence only a low volume fraction of the shells. After the final centrifugation a surplus of solvent was almost absent inside the capillary, as the pure solvent leaked through the wax. However, we found regions of high volume fraction of shells opposite to the sediment-wax interface. These high density regions perhaps resulted from the drainage of solvent and subsequent compression by capillary action. The height of the sediment in this capillary was approximately 14 $\mathrm{mm}$.

Another capillary, capillary 3 (dimensions same as capillary 1) was prepared with a batch of shells of particle radius $R_{\mathrm{t}}=2.02$ $\pm 0.03 \mu \mathrm{m}$ and shell thickness $d \approx 90 \mathrm{~nm}$ (both measured from SLS) with $d / R_{\mathrm{t}}=0.04$. The sample was centrifuged at $2000 \mathrm{~g}$ for 10 min to obtain compressed shells.

\subsection{Image acquisition}

3D image stacks of packings of shells at different volume fractions were acquired from different heights in the sediment, in the capillaries 1 and 2 using a Nikon C1 confocal microscope with a $63 \times$ NA 1.4 oil immersion objective (Nikon) in 
fluorescence mode. From capillary 3 only one volume fraction was taken. The capillary was mounted on a microscope stage tilted 90 degrees with respect to gravity. This way the capillary could remain in an upright position so as not to disturb the packing. The first image stack was taken $5 \mathrm{~h}$ after centrifugation. Most of the image stacks, except the lower packing fractions were acquired within a duration of 1-3 days after sample preparation. During this period we didn't observe a noticeable relaxation of the shells in the sediment. The low density regions in the capillary were scanned randomly at various times within a period of 1 month. The capillary was kept undisturbed during this period. The height of the sediment was measured to be nearly the same $(7 \mathrm{~mm})$ as the original height during this period.

The RITC dye in the shell was excited with a $543 \mathrm{~nm}$ HeNe laser. The sample was scanned with a pixel dwell time of 3.12 $\mu \mathrm{s}$ and a frame rate of $0.196 \mathrm{fps}$. While scanning in the $Z$ direction the laser power was increased typically from $4-7 \%$ to $10-12 \%$ of total power. We did this to compensate for the intensity drop deep in the sample caused by the index mismatch between the sample $\left(n_{\mathrm{D}}^{20}=1.42\right)$ and the immersion medium $\left(n_{\mathrm{D}}^{20}=1.5\right)$, causing some scattering and a decrease of the point spread function with depth. ${ }^{58}$ An image stack comprised of $1024 \times 1024 \times 790$ voxels with a voxel size of $0.0976 \times 0.0976 \times 0.1094 \mu \mathrm{m}^{3}$, giving a total 3D image volume of $99.94 \times 99.94 \times 86.43 \mu^{3}$. Acquisition of this image stack approximately took $67 \mathrm{~min}$. The voxel size in $Z$ was corrected for index mismatch with a scaling factor $0.91 .^{58,59}$ During scanning there was no drift in the suspension and the particles were tightly packed such that they did not undergo Brownian motion.

\subsection{Bond order analysis}

The bond orientational order parameters we calculated for each particle $i$ are defined by a complex vector,

$$
q_{l m}(i)=\frac{1}{N_{\mathrm{b}}(i)} \sum_{j=1}^{N_{\mathrm{b}}(i)} Y_{l m}\left(r_{i j}\right)
$$

where $N_{\mathrm{b}}(i)$ is the number of nearest neighbors of particle $i, r_{i j}$ is the vector from the center of particle $i$ to particle $j$ and $Y_{l m}$ are the spherical harmonics (with $m=-l$ to $m=+l$ ). The sum runs over all neighbors of particle $i$. Neighbors are usually defined as all particles that are within a given cut off distance around a particle. To distinguish between a disordered and a crystalline environment of a particle $i$ from these sets of bond orientational order parameters, we calculated both the local bond order parameter (Steinhardt order parameter) $q_{l}(i)^{54}$ and the average of bond orientational order parameters over particle $i$ and all its neighbors i.e. $\bar{q}_{l}(i)$, following the method of Lechner and Dellago. ${ }^{55}$ The distributions of the averaged local bond order parameter are narrower because of the averaging and the fact that it takes into account the second shell of neighbors, whereas the Steinhardt order parameter is limited to the first shell. Hence, this makes it also suitable for identifying the type of crystal structure (if any).
The Steinhardt local bond order parameter is,

$$
q_{l}(i)=\sqrt{\frac{4 \pi}{2 l+1} \sum_{m=-l}^{l} q_{l m}(i)^{2}}
$$

and its averaged form is,

$$
\bar{q}_{l}(i)=\sqrt{\frac{4 \pi}{2 l+1} \sum_{m=-l}^{l}\left|\bar{q}_{l m}(i)\right|^{2}}
$$

where,

$$
\bar{q}_{l m}(i)=\frac{1}{N_{\mathrm{b}}(i)+1} \sum_{j=0}^{N_{\mathrm{b}}(i)} q_{l m}(j)
$$

here, the sum runs over all neighbors of particle $i$ plus the particle $i$ itself. The cut off distance that we set for the present system was taken to be the position of the first minimum in the $g(r)(r / D=1.24)$

\section{Results and discussion}

\subsection{Reversible elastic deformation of jammed shells}

The elastic shells in jammed packings at higher densities are deformed such that at each contact one of the shells buckles with a dimple and the other remain spherical. We checked the reversibility of deformation of shells in a strongly compressed packing by releasing the stress acting on individual particles. The pressure was released by rotating a sample capillary, which contained a sediment of height around $5 \mathrm{~mm}$, from its initial upright position to a horizontal position. This resulted in the expansion of the sediment in the direction perpendicular to gravity. Fig. 2A is a confocal image taken from a region close to the bottom of the sediment. In the right half of the image shells

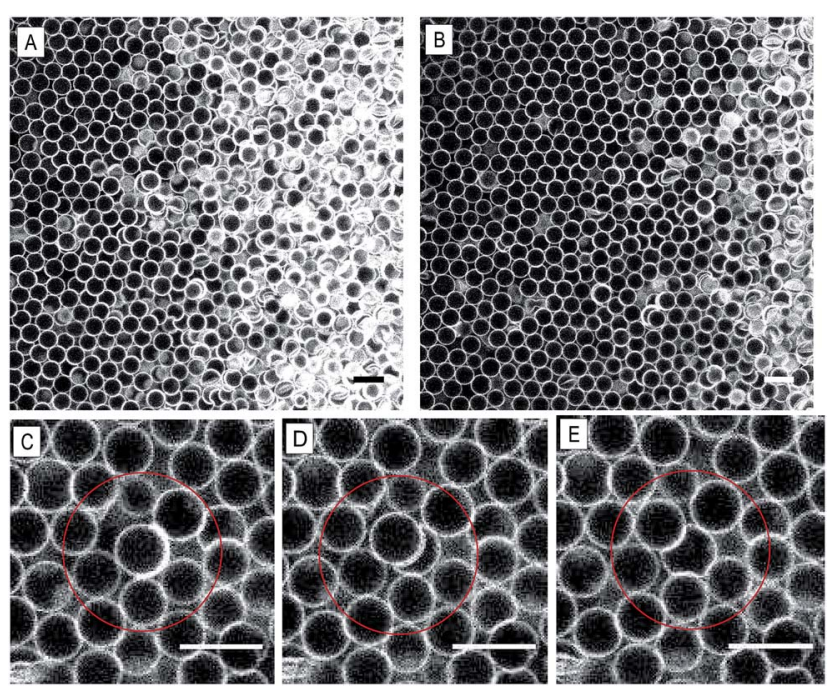

Fig. 2 Confocal images of compressed shells upon release of stress. (A) Shells in the sediment close to the wax interface. (B) Same spot taken 40 min later. Elastic recovery of a highly deformed shell from a bowl shape to almost spherical shape at (C) $0 \mathrm{~s}$, (D) $38 \mathrm{~s}$ and (E) $76 \mathrm{~s}$. All the scale bar represent $10 \mu \mathrm{m}$. 
are still highly deformed and some are even hard to recognize individually, while on the left the shells are almost spherical again. Image $2 \mathrm{~B}$ is the same area after $40 \mathrm{~min}$. The number of highly deformed shells is clearly less in this image due to the expansion of the sediment and the resulting drift of the expanding shells to the left. While the packing of shells was expanding they also completely recovered their initial spherical shape. Fig. 2C-E are zoomed in confocal pictures that focus on the elastic recovery of an extremely deformed, almost completely emptied, shell during stress release. In Fig. $2 \mathrm{C}$ this shell can be seen as a thick white hemispherical segment running along the contour of a spherical particle (in the center of the red circle), with an overlap depth $\delta_{i j} \approx\left(R_{i}+R_{j}\right) .38 \mathrm{~s}$ later the overlap depth was reduced by $43 \%$ and subsequently by $78 \%$ from the initial depth as shown in Fig. 2D and E. Here the applied stress from its contact neighbor was released when the neighbor was pushed to an adjacent void space. This recovery from even extreme deformation is a clear indication that the shells can reversibly sustain large forces and that the interaction between the shells is purely repulsive. Hence this system is a suitable model soft frictionless granular system for jammed matter studies.

\subsection{Image processing}

Fig. 3A-C shows confocal images (16 bit) of $X Y, Y Z$ and $X Z$ slices out of a full $3 \mathrm{D}$ data stack. The image quality is good enough, even in $Z$, to perform a quantitative structure analysis. It can be seen that every particle deforms in a rather regular way: the overall shape remains spherical, but at the points of contact with a neighbor the particle either received or produced a spherical indentation from/in its neighbors (Fig. 3D). It is this

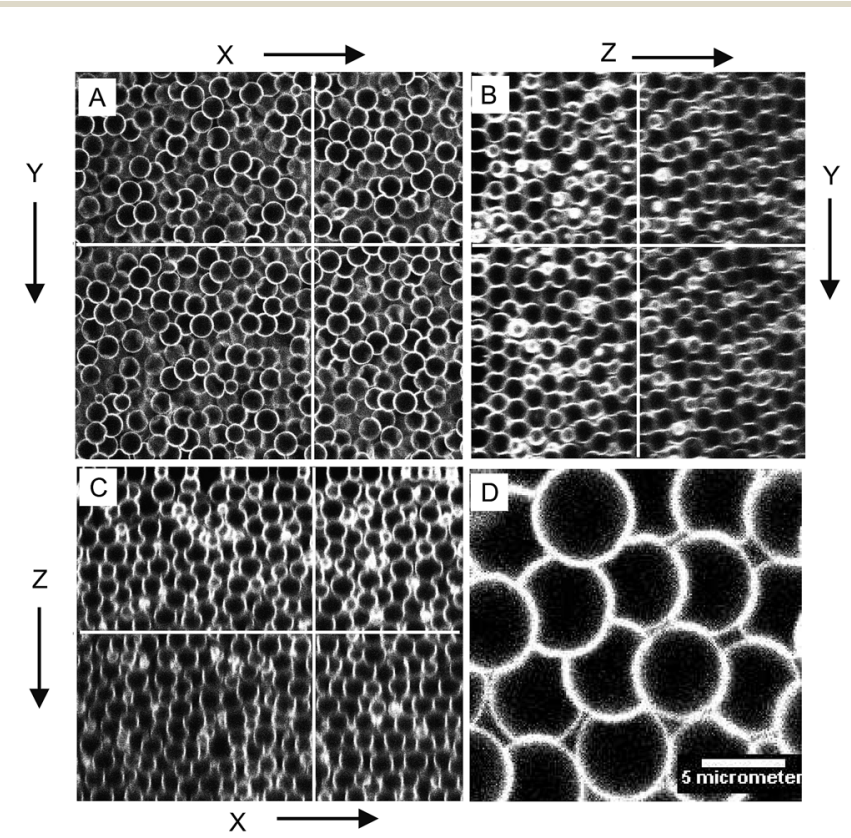

Fig. 3 (A) Confocal microscopy images of a dense packing of fluorescent shells in an $X Y,(B) Y Z$ and (C) $X Z$ slice. Image size in $X Y$ is $1024 \times 1024$ pixels and 790 slices in $Z$. (D) Zoomed in picture of deformed shells. regular shape-deformation that allows us to determine welldefined particle centers and hence parameters characterizing the amorphous structure. The image processing routine we have developed to find the positions of the shells in 3D consists of three steps: locating the edges of the individual particles distributed over several consecutive slices, analyzing their shapes in each slice to obtain their $2 \mathrm{D}$ centers and radii, and finally grouping their radii and centers to reconstruct the particles in 3D. The image processing was implemented using the free image processing software package Image J. ${ }^{51}$

The first three steps in image analysis are described in sections 3.2.1 to 3.2.3. Finally, in section 3.2.4 we describe a method to identify the dimpled or deformed particle in each contact pair.

3.2.1 Edge detection. In dense packings of elastic shells, the shells are in close contact with their neighbors and even undergo deformations at contact points. Fig. 4A shows a part of a $2 \mathrm{D}$ confocal image from a $3 \mathrm{D}$ stack of compressed shells. In the image the brightest pixels correspond to the fluorescence intensity from the shell region of the particles. These pixels form contours that delineate the shapes of the deformed particles. The black region enclosed within each white contour and in the space between particles corresponds to the index matching solvent mixture (DMSO + ethanol). The width of the white contour cannot be directly compared to the thickness of the shell, or even of two shells in direct contact, because the actual shell thickness $(56 \mathrm{~nm})$ is well below the lateral resolution of the microscope $(\sim 200 \mathrm{~nm})$. But a difference in the contour width and in brightness is visible in regions where the particles are in contact compared to the non-contact regions. At the contact regions the contour is wider and brighter due to the double contribution to the fluorescence intensity from both the particles in a pair. Besides, in the image the contrast between the shell region and the interior is not the same for each particle. This is caused by a convolution of the thin shell and the

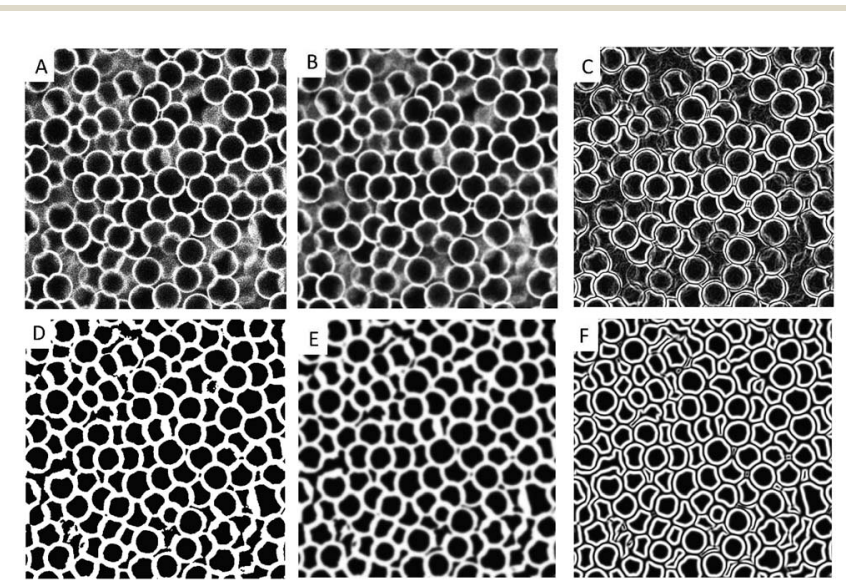

Fig. 4 Different stages of edge detection: (A) 2D confocal image of hollow fluorescent shells. (B) Gaussian smoothing, $\sigma=1.5$ pixels, (C) edges detected with the Sobel algorithm directly on image $B,(D)$ binary image after local thresholding of image $B$, (E) greyscale image obtained by convolution of the binary image with a Gaussian, $\sigma=1.5$ pixels, and (F) edges detected with the Sobel algorithm in image $E$. Image size is $544 \times 544$ pixels. 


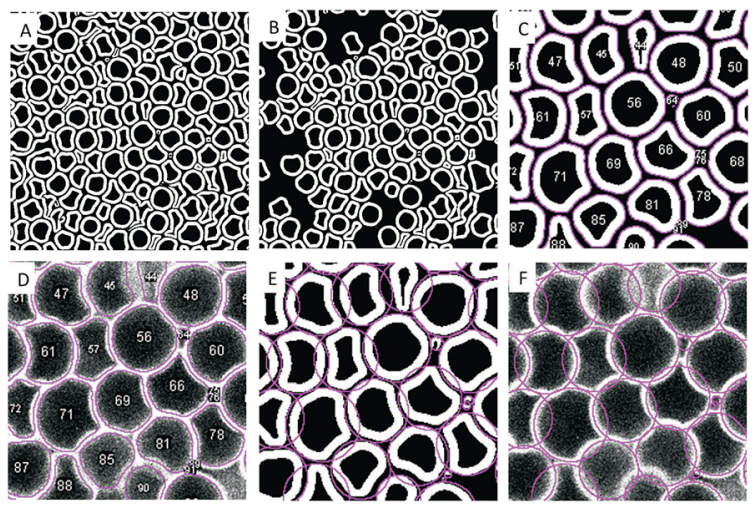

Fig. 5 Different stages of shape determination: (A) binary image of particles obtained from the edge detection step, (B) particles detected using the Analyze Particles algorithm, (C) zoomed in view of image $B$ with particles labeled and highlighted, (D) overlay of the original confocal image and the detected particles, $(E)$ the detected particles enclosed by the SEC'S and (F) overlay of the original image and the SEC's that enclose particles. Image size of A and B are $544 \times 544$ pixels whereas C, D, E and F are $210 \times 210$ pixels. The pixel size is $0.0976 \mu \mathrm{m}$ in all images. non-uniform brightness of white contours and poor contrast in some particles. We found that applying the Sobel algorithm directly on a smoothed image resulted in broken edges for some particles, as seen in Fig. 4C. The edge is broken at regions were the particles had poor contrast. This will result in missing of shells in 2D planes, which is not desirable. To overcome this problem we first applied a threshold to the greyvalue of pixels in the smoothed image. The value of the threshold for each pixel was decided locally by a local thresholding algorithm. ${ }^{61}$ Among the local thresholding algorithms we found the mean algorithm to work best in these images. With this algorithm, each pixel is set at a threshold value which is equal to the mean of the greyvalue of its neighbors within a window of size 25 pixels, approximately the radius of the particle. This resulted in a binary image shown in Fig. 4D. In the third step the binary image was converted back to greyscale by convolving it with a gaussian function of $\sigma=3$ pixels, Fig. 4E. The greyscale image after thresholding and convolution clearly has a much higher contrast and more uniform brightness in the shell regions compared to the one before, Fig. $4 \mathrm{~B}$. We did this step to make the profile of the intensity near the shell smoother. In the final step we applied the Sobel algorithm. Fig. $4 \mathrm{~F}$ shows the gradient image of particles separated from their neighbors by edges represented by white pixels. The image Fig. $4 \mathrm{~F}$ also contains features enclosed by edges that do not resemble particles in regions where they are barely visible. These erroneous features were removed in later stages of the image processing.

3.2.2 Shape determination and fitting. The second step in finding the positions of the particles includes analyzing the shape of the individual shells bounded by their edges in Fig. 4F. In ImageJ there is an algorithm called Analyze Particles ${ }^{51}$ which counts and measures objects in binary or thresholded images. It works by scanning the image until it finds the edge of an object. By applying this algorithm on the binary image, Fig. 5A (obtained by the binarization of gradient image Fig. 4F from edge detection) we obtained Fig. 5B. Note that during this step objects close to the edge of the image volume did not have a closed contour and were therefore not detected. To remove erroneous objects that do not resemble the particles in Fig. 5A we limited the circularity of the objects to 0.2-1.0. Objects outside this limit mainly consisted of out-of-focus particles that appear fused together in the original image. This creates voids or black regions in the image, which does not mean that particles are missing from our analysis. These particles will be properly identified in subsequent slices in the stack. Any remaining erroneous objects were removed in a later step of the analysis. Fig. 5C shows the zoomed in $(210 \times 210$ pixels $)$ view of objects highlighted and marked with numbers.

To check how well the edge and the shape of the detected particles match with the original particles, we overlayed the processed image with the original image as shown in Fig. 5D. The shapes of the detected particles closely resemble the actual shapes of the particles. However, the detected particles are not touching each other anymore, though the shells were in contact in the original image. The image processing has introduced a gap of a few pixels between neighboring particles. For shape analysis we ignored these pixels, but we took them into account in the final stage of finding the positions and radii of the particles.

Furthermore, since the shells were spherical initially before compression, the geometric $2 \mathrm{D}$ center of the detected particles in each slice does not correspond to the center of the original shells in that slice. Instead, we determined the 2D center of the original particle in each slice by fitting the smallest circle that still encloses the detected particle shape as shown in Fig. 5E. We used the algorithm called Smallest Enclosing Circle (SEC) ${ }^{51}$ in ImageJ for fitting the circle. The algorithm takes all the $X Y$ coordinates in the perimeter of the detected particle and calculates iteratively the geometric center and radius of the circle that includes the farthest coordinate from the center. Fig. $5 \mathrm{~F}$ shows the overlay of the original image and the corresponding SEC's. The perimeter of the circles run almost exactly 
through the convex contours of the original particles. The overlap areas of the circles correspond to the area of the dimples.

3.2.3 Centroid and radius determination. From the shape determination step we obtained the radii and centers of the SEC's that enclose the detected particles in all 2D slices in a 3D stack. Fig. 6A shows the probability distribution of radii of the SEC's, $R_{\mathrm{SEC}}$, from the stack of image volume $99.94 \times 99.94 \times$ $86.43 \mu \mathrm{m}^{3}$, after filtering out the rest of the erroneous objects, based on size, that were not removed in the shape determination stage. These objects mostly consist of interstitial spaces between the shells. Here we also compensate for the gap between the detected particles in each slice by increasing the radii of the SEC's by half the width of the gap, which was 2 pixels in the image. The peak of the distribution that occurs at around 29 pixels, close to the measured radius of the original particles, which was 28 pixels. The tail of the distribution reflects the radii of cross-sections away from the diameter of the particles and also the presence of a few large particles in the packing.

In the final stage of locating particle positions we grouped together the SEC's that belong to the same particle and reconstructed the actual particle. We grouped the SEC's by searching for similar $X Y$ centers within a defined interval in $Z$. The interval in $Z$ was set at a value less than the diameter of the particle, as the particles were not nicely detected near the poles. For these particles of diameter 50 pixels in $Z$ we set the interval at 35 pixels. To reconstruct the original particle from the collection of typically around 25 SEC's obtained for each particle, we considered the plot of the radii of the SEC's against their corresponding $Z$ position in the stack. An example is shown in Fig. 6B. These data points were then fitted with the equation of a circle, $R_{\mathrm{SEC}}{ }^{2}=R_{\mathrm{t}}{ }^{2}-\left(Z-Z_{\mathrm{c}}\right)^{2}$ (Fig. 6B). The fit gives the center $Z_{\mathrm{c}}$ and the total radius of the particle, $R_{\mathrm{t}}$. The $X$ and $Y$ coordinates of the center of the particle were obtained by taking the average of $X$ and $Y^{\prime}$ 's of the set of SEC's that make up each particle. The average of the standard deviations in the center coordinates $X_{\mathrm{c}}$ and $Y_{\mathrm{c}}$ was $80 \mathrm{~nm}$ and in $Z_{\mathrm{c}}$ it was $73 \mathrm{~nm}$ (estimated from the fit), which are below the pixel size. The slightly higher value of error in $X_{\mathrm{c}}$ and $Y_{\mathrm{c}}$ compared to $Z_{\mathrm{c}}$ most likely originated from the slight inaccuracies involved in the shape determination and fitting of SEC's. For some particles we found that the $Z_{\mathrm{c}}$ deviated from the actual center. This was caused by the overlap of SEC's from particles lying exactly on top of one another and also from the contribution of SEC's of any remaining erroneous objects. We sorted out these ambiguous particles by setting a threshold for the standard error ( $>90 \mathrm{~nm}$ ) in $Z_{\mathrm{c}}$ obtained from the fit. A typical graph of such an ambiguous particle with incorrect $Z_{\mathrm{c}}$ is shown in the ESI (Fig. S2(A) $\dagger$ ). Apart from ambiguous particles $(<2 \%)$, the fit gave good results for all the particles irrespective of their deformed/buckled shape or the number of dimples they posses (ESI Fig. S2(B-D) † shows the fit for particles with 2, 6 and 10 dimples, where the number of dimples is measured by a procedure described else where in this article). This is due to the fact that the deformed shape in each slice is fitted by a smallest circle (resembling spherical shape of shell) that encloses the particle shape.

We identified 5753 particles in a volume of $99.94 \times 99.94 \times$ $66 \mu^{3}$. This number (5753) does not include the particles close to the edge in the $X$ and $Y$ direction as they were not identified during image analysis. Note that a particle near the edge is only identified if its full volume resides inside the image volume. We also excluded about four layers of particles in the $Z$ direction due to slight ordering near the wall. Fig. 6C shows the distribution of radii $R_{\mathrm{t}}$ of the identified shells. This distribution is
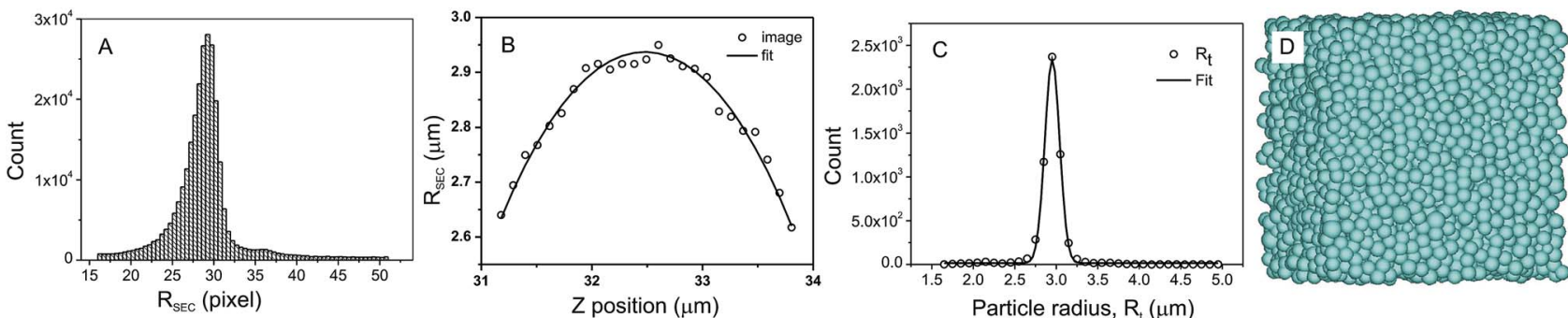

Particle radius, $\mathrm{R}_{\mathrm{t}}(\mu \mathrm{m})$
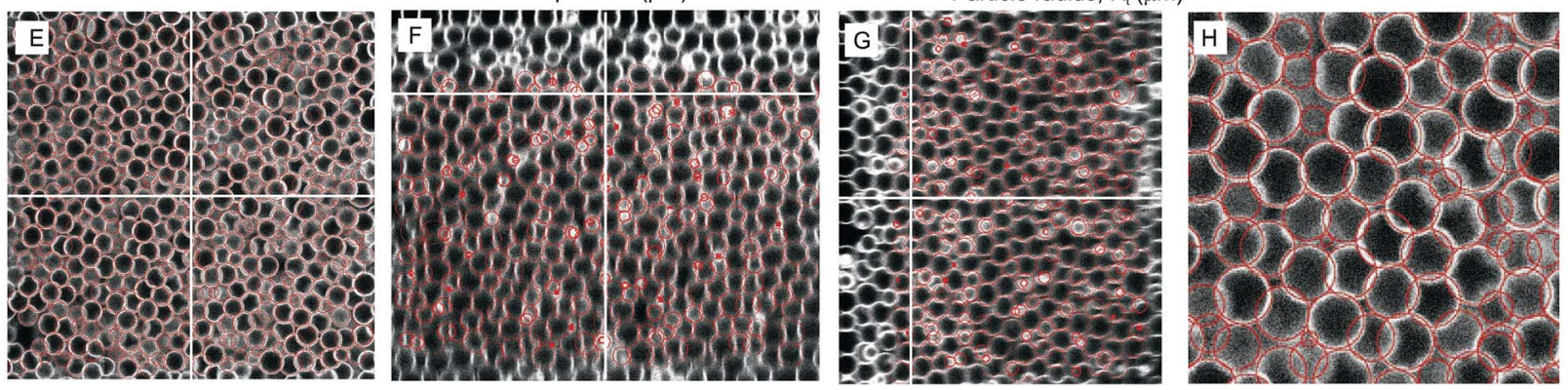

Fig. 6 (A) Distribution of radii of the smallest enclosing circles (SEC's), $R_{\mathrm{SEC}}$ enclosing the detected particles in each slice in a 3D stack of images. (B) Plot of $R_{\mathrm{SEC}}$ VS. corresponding $Z$ position of a particle. The line is the best fit of a circle through the data points with fit parameters: $Z_{\mathrm{C}}=32.48 \pm$ $0.01 \mu \mathrm{m}$ and radius of the particle $R_{\mathrm{t}}=2.937 \pm 0.002 \mu \mathrm{m}$. (C) Distribution of the particles radii $R_{\mathrm{t}}$. We measured an average radius of $2.95 \mu \mathrm{m}$ with a standard deviation of $0.17 \mu \mathrm{m}$ obtained from a Gaussian fit (line) (D) 3D reconstruction of particles in an image volume of $99.94 \times 99.94 \times 66$ $\mu \mathrm{m}^{3}$ at a volume fraction of 0.908 . The reconstructed spheres (shown in red) overlayed on original confocal images in a (E) $X Y$, (F) $X Z$ and (G) $Y Z$ slice (image size is $99.94 \times 99.94 \mu \mathrm{m}$ in $X Y$ and $86.43 \mu \mathrm{m}$ in Z). (H) A zoomed in view of overlay image (E). 
nicely symmetric with average particle radius $R_{\mathrm{t}}=2.95 \mu \mathrm{m}$ and a standard deviation $0.17 \mu \mathrm{m}$, this corresponds to $6 \%$ polydispersity. The radius of the particles obtained from image analysis is close to the value that was obtained from SLS, which is more accurate, but polydispersity is slightly higher resulting from the inaccuracies in image analysis. In these 5753 particles there were $1.4 \%$ particles with a wrong value of $Z_{\mathrm{c}}$, which is a relatively small fraction. These particles along with the small fraction of small particles $(1.8 \%)$ and large particles contribute to the flat tail of the distribution of $R_{\mathrm{t}}$. Fig. 6D shows the 3D reconstruction of the packing containing 5753 particles. In addition, the reconstructed particles were overlayed on the original confocal images to examine the accuracy of the algorithm in finding the positions and radii of shells as shown in Fig. 6E-H. The circles (shown in red) depicts the identified particles in $X Y, X Z$ and $Y Z$ slices of the confocal image of image size $99.94 \times 99.94 \mu \mathrm{m}$ in $X Y$ and $86.43 \mu \mathrm{m}$ in $Z$. Clearly, all shells are identified in the image volume, except the shells near the edge in $X Y$ and four layers (close to the capillary wall) in $Z$ direction, which are anyway not included in the analysis. The circles even pass through the convex thin white bands that correspond to the shell boundaries validating the accuracy with which radii are determined using our algorithm. Finally a check for pixel biasing ${ }^{39}$ (ESI Fig. S3†) showed a nearly flat histogram of the fractional part of particle coordinates $X_{\mathrm{c}}, Y_{\mathrm{c}}$ and $Z_{\mathrm{c}}$ indicating that the particles are located with sub-pixel resolution. However, we did see a strong bias in the fractional part of the 2D centers, $X_{\mathrm{SEC}}$ and $Y_{\mathrm{SEC}}$ at 0 and 0.5 (not shown). This could also be the reason for the slightly higher value of the error in $X_{\mathrm{c}}$ and $Y_{\mathrm{c}}$ compared to $Z_{\mathrm{c}}$.

The volume fraction of this packing was 0.908 . This value was obtained by summing the volumes of 5753 spheres and subtracting their overlap volume in an image volume of (99.94 $\left.-2 R_{\mathrm{t}}\right) \times\left(99.94-2 R_{\mathrm{t}}\right) \times 66 \mu \mathrm{m}^{3}$. The image size was reduced by $R_{\mathrm{t}}$ from all sides in the $X$ and $Y$ directions to compensate for the particles that are missing near the edge. Note that we also included the small fraction of ambiguous particles in volume fraction calculation as they also represent a particle, but with a higher error in $Z$ coordinate. For particles straddling the edges of the reduced volume it somewhat arbitrary how much of their volume should be included in the volume fraction measurement. This produced to an uncertainty of about 0.005 in the determination of the volume fraction. But the value of volume fraction is highly sensitive to the radius of the particles obtained from the image analysis. Therefore, the absolute volume fraction may contain a systematic error of about 0.04 . But the error in comparing samples at different volume fractions will be only about 0.005 . Table 1 gives an overview of each packing at different volume fractions obtained from all the capillaries. The apparent clustering of data points around a volume fraction of 0.9 reflects the fact that upon further compression the volume squeezed out of the system apparently comes only from a reduction of shell volumes rather than a reduction of interstitial space, leaving the volume fraction unchanged.

3.2.4 Dimple detection. Knowing the coordinates of the particles, we proceeded to identify the particle in contact pairs

Table 1 The details of image stacks of different densities obtained from image analysis algorithms from capillaries 1 and 2, containing shells of $d /$ $R_{\mathrm{t}}=0.02$. Stacks highlighted by * were taken from capillary 2 and the image stack T1 corresponds to sample of shells of $d / R_{\mathrm{t}}=0.04$ (capillary 3 )

\begin{tabular}{|c|c|c|c|c|c|c|}
\hline Image stack & No. of particles & Ambiguous particles (\%) & No. of contacts & $R_{\mathrm{t}}(\mu \mathrm{m})$ & $\varphi$ & Rattlers (\%) \\
\hline $\mathrm{S} 1 *$ & 6417 & 1.3 & 29117 & 2.928 & 0.892 & 0.0 \\
\hline $\mathrm{S} 2 *$ & 6093 & 1.85 & 28210 & 2.926 & 0.913 & 0.0 \\
\hline $\mathrm{S} 3 *$ & 5955 & 1.9 & 26682 & 2.958 & 0.900 & 0.0 \\
\hline $\mathrm{S} 4 *$ & 5844 & 1.3 & 25473 & 2.956 & 0.895 & 0.0 \\
\hline S5* & 5753 & 1.4 & 26131 & 2.952 & 0.908 & 0.0 \\
\hline $\mathrm{T} 1$ & 3810 & 1.2 & 17774 & 2.171 & 0.906 & 0.0 \\
\hline S6* & 5737 & 1.8 & 25084 & 2.962 & 0.897 & 0.0 \\
\hline S7 & 5307 & 1.65 & 24368 & 3.002 & 0.888 & 0.0 \\
\hline S8 & 5163 & 1.16 & 22996 & 2.991 & 0.883 & 0.0 \\
\hline S9* & 4964 & 1.2 & 21094 & 2.962 & 0.852 & 0.0 \\
\hline S10* & 4714 & 0.9 & 19581 & 2.954 & 0.835 & 0.0 \\
\hline S11 & 4570 & 0.37 & 18154 & 2.967 & 0.806 & 0.0 \\
\hline $\mathrm{S} 12^{*}$ & 4552 & 0.8 & 18522 & 2.948 & 0.810 & 0.0 \\
\hline S13 & 4475 & 1 & 17751 & 2.948 & 0.793 & 0.0 \\
\hline S14 & 4339 & 0.25 & 17113 & 2.968 & 0.791 & 0.0 \\
\hline S15 & 4294 & 1 & 16178 & 2.942 & 0.765 & 0.0 \\
\hline S16 & 4264 & 0.9 & 15694 & 2.938 & 0.763 & 0.0 \\
\hline S17 & 4254 & 0.18 & 16388 & 2.967 & 0.776 & 0.0 \\
\hline S18 & 4196 & 0.7 & 14992 & 2.937 & 0.752 & 0.1 \\
\hline S19 & 4084 & 0.6 & 13722 & 2.926 & 0.726 & 0.7 \\
\hline $\mathrm{S} 20$ & 4030 & 0.39 & 12985 & 2.929 & 0.721 & 1.1 \\
\hline S21 & 4024 & 0.54 & 13691 & 2.934 & 0.723 & 0.5 \\
\hline S22 & 3913 & 0.3 & 11903 & 2.922 & 0.701 & 2.1 \\
\hline S23 & 3889 & 0.35 & 11733 & 2.925 & 0.699 & 3.4 \\
\hline $\mathrm{S} 24$ & 3933 & 0.7 & 11375 & 2.930 & 0.693 & 3.5 \\
\hline S25 & 3828 & 0.47 & 10380 & 2.919 & 0.677 & 4.9 \\
\hline S26 & 3647 & 0.13 & 8495 & 2.933 & 0.663 & 16.0 \\
\hline
\end{tabular}




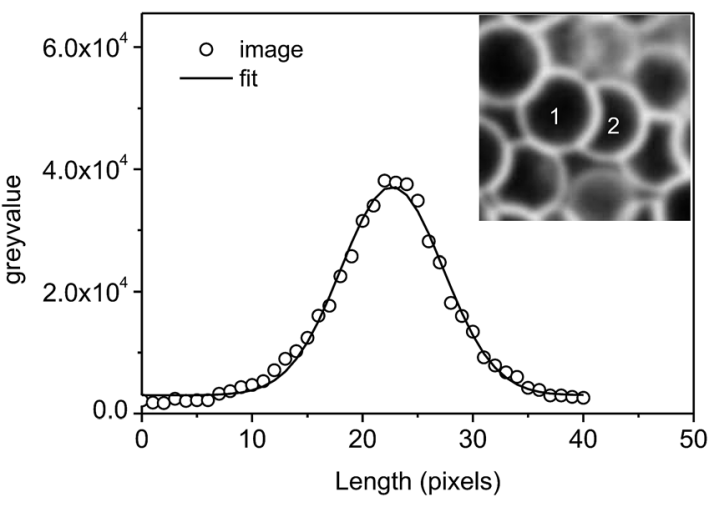

Fig. 7 The greyvalue distribution of pixels that make up the line of length 40 pixels, joining the 3D centers of particles 1 and 2, shown in the inset confocal image. The line in the graph is a Gaussian fit with peak center at 22.69 pixels.

that had a dimple. The dimpled particle was detected by drawing a line that connects the $3 \mathrm{D}$ centers of neighboring particles and then locating the position of the maximum in the fluorescence intensity along the line. The position of the maximum should be closer to the center of the particle that is dimpled. The lines that connect 3D centers of pairs of particles in a stack were drawn by the 3D Bresenham algorithm. ${ }^{62}$ This algorithm is widely used to draw pixelated lines connecting two end points in a digital image. The algorithm takes the 3D coordinates of the centers in a pair of particles as the end points and generates intermediate coordinates iteratively. Out of the three coordinates $X_{\mathrm{C}}, Y_{\mathrm{C}}$ and $Z_{\mathrm{C}}$ of the particle, one coordinate is decided by the slope of the line. The remaining two are determined by a decision variable that decides on one of the two choices for the next pixel in a line based on the relative positions of the true line and the midpoints of the pixels. Before applying the algorithm the original confocal images in the stack were smoothed by a Gaussian blur filter of $\sigma=3$ pixels. Fig. 7B represents the greyvalue or intensity profile taken along the line that joins the 3D centers of particles marked 1 and 2 in the inset confocal image, taken from a packing of volume fraction 0.908 . In the profile circles denote the greyvalues of the pixels along the line connecting the centers, as obtained from the image. The line is a Gaussian fit. The position of the center of the peak is found to be closer to particle 2 and hence particle 2 is the correctly identified dimpled particle. Similarly we identified the dimpled particles in all contact pairs in the whole packing. Out of 26131 contact pairs in this packing only $0.19 \%$ resulted in a wrong peak center and 4 pairs with a position of the peak at equal distance from the center of particles in a pair.

\subsection{Microstructure of compressed shells}

3.3.1 Radial distribution function $g(r)$. From the particle coordinates we calculated the radial distribution function $g(r)$, which is the probability of finding a particle at a distance $r$ from a given reference particle. ${ }^{63}$ Fig. 8 shows $g(r)$ for shells $\left(d / R_{\mathrm{t}}=\right.$ 0.02 ) compressed at different volume fractions $\varphi$. The shape of the distribution resembles the structure of a liquid, but was less well-defined than that of a hard sphere packing at the same $\varphi$,
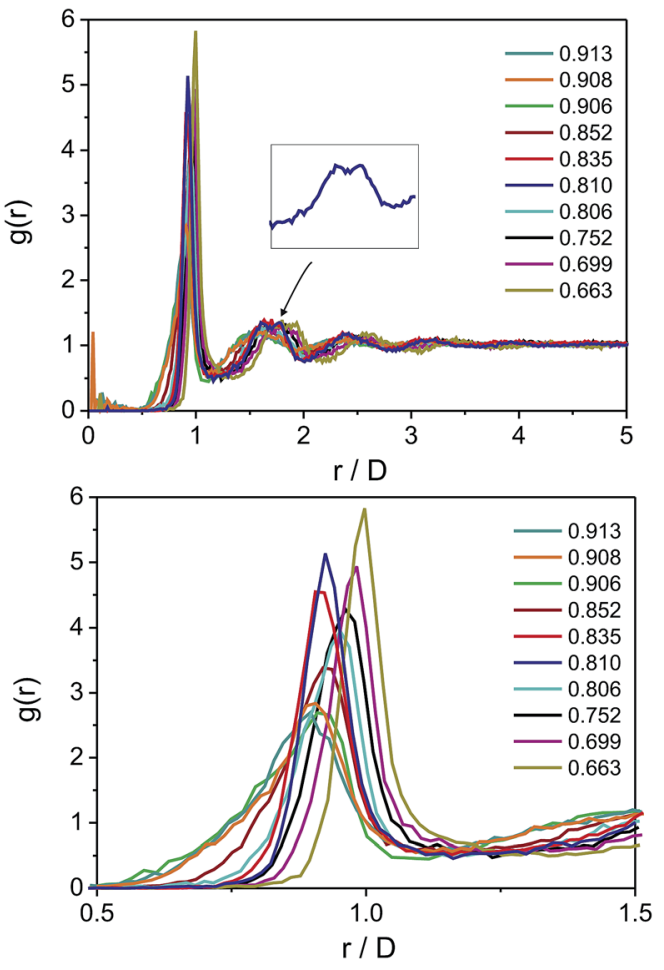

Fig. 8 Radial distribution functions of compressed deformable shells spanning the range of experimental volume fractions. The radial distances for each packing is normalized by the corresponding average particle diameter obtained from image analysis. Inset figure shows the split second peak of the $\varphi=0.810$ packing. Volume fraction $\varphi=0.906$ represents the shells with shell thickness to diameter ratio, $d / R_{\mathrm{t}}=0.04$. The bin size is 0.1 .

except for the lowest volume fraction which is close to random close packing (RCP). ${ }^{40,64}$ Besides, the amplitude of the peaks decayed and the width of the peaks increased with increasing $\varphi$. This behavior is just opposite to that of liquids, which have $\varphi$ lower than the jamming point. The $g(r)$ of the $\varphi=0.663$ peaks at $r=D(D$ is the diameter of particle), confirming that this packing is close to the jamming point. The full width half maximum of this peak is $0.08 D$, which is nearly equal to the sum of the polydispersity $(0.06 D)$ in radii of the particles and the error in the determination of the distance between the centers of the two particles $(2 \times 80 \mathrm{~nm}$, or $0.027 D)$. Moreover, the first peak of $g(r)$ of the denser packings occurred at radial distances, $r / D \leq 1$ and also shifted to even smaller distances as the volume fraction is increased in the packing. The shift and broadening of the first peak upon increasing the density resulted from the increase in deformation of the shells by their contacting neighbors. Similar behavior of $g(r)$ was predicted in simulations on soft granular particles. ${ }^{4,65,66}$ The small sharp peaks near $r / D$ $\approx 0$ are the contributions from the small fraction of incorrectly identified (false) particles $(<2 \%)$ by our image analysis procedures (Table 1). For two of the packings, the one at $\varphi=0.810$ and another at $\varphi=0.835$, the position and width of the first peak of $g(r)$ do not fall in the trend displayed by the other samples. Besides, $\varphi=0.810$ showed a split second peak (shown also in the inset) which was not seen in $\varphi=0.835$. The splitting 
of second peak has been reported for hard-sphere glasses, ${ }^{67-69}$ and is known to occur close to the glass transition, which is not the case here. In a recent simulation by Silbert et al. ${ }^{66}$ a similar split second peak was observed in $g(r)$ for a zero-temperature, disordered, monodisperse soft-sphere packing at the jamming/ unjamming transition. However, this split second peak has not been observed in experimental jammed systems of monodisperse spheres, for example in monodisperse soft colloidal particles made from a thermosensitive hydrogel. ${ }^{70}$ The splitting in the second peak was reported to be a likely indication of local order in the particle positions. ${ }^{69}$ But, upon visualization of the 3D image stack we did not see a local crystalline order in this packing. So to quantitatively measure the degree of local symmetry present in these packings and compare it to others, we performed a local bond order calculation as described in the experimental method section. The results of local bond order parameter $q_{6}$ (dashed lines) and its averaged form $\bar{q}_{6}$ (solid lines) are shown in the Fig. 9. The values of the order parameter tend to be higher (with peak of the distribution at values greater than 0.4) when the local order resembles a bcc or fcc crystalline structure and the values are lower (position of peak at values less than 0.4) when the local environment is a glass or fluid-like reported for systems where the particles interact via a LennardJones potential and one in which the interaction is of the Gaussian core form. ${ }^{55}$ As expected, the distribution of $q_{6}$ is relatively broad compared to $\bar{q}_{6}$, making it difficult to distinguish between amorphous and crystalline structures, but from both distributions it seems like there is little or no order in these packings. Besides, the distribution for various volume fractions are well distinguishable from one another and shift to lower $q$ values with increasing volume fraction, indicating that the packings became more disordered at higher compression. At the jamming point the overlap between all particles simultaneously goes to zero. So the distribution of distances is the most uniform at this point. Apparently this also gives large bond order. In fact, this time all the volume fractions follow the same trend (in both $q_{6}$ and $\bar{q}_{6}$ ), including $\varphi=0.810$ and $\varphi=$ 0.835 that showed a different behavior in $g(r)$ that pointed towards a more crystalline order compared to other packings.

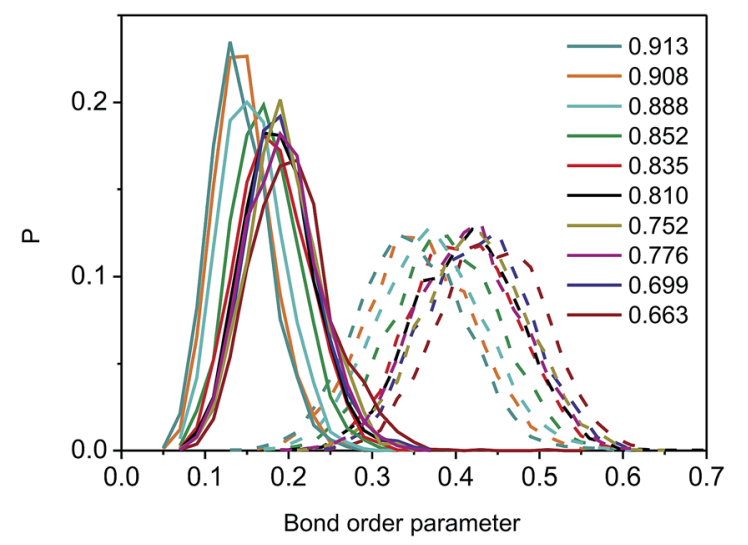

Fig. 9 Probability distributions of $q_{6}$ (dashed lines) and $\bar{q}_{6}$ (solid lines) values in packings of elastic shells over a range of volume fractions. The bin size is 0.02 .
But, we could not confirm this crystalline order by a local bond order analysis or by a visual inspection.

3.3.2 Contact number $(Z)$ and buckling number $\left(N_{\delta}\right)$. Another parameter that describes the structure of an amorphous packing is the number of contacts per particle $Z$. We determined the number of contacts for each shell from the coordinates of their centers and radii. A pair of particles is considered a contact pair if the distance between their centers is less than or equal to the sum of their radii. The chances of missing a contact in our system are determined by the resolution in finding the radii and particle position (which is less than one pixel, about $80 \mathrm{~nm}$ ). The distributions of the contact number $Z$ for a several samples at different volume fractions are shown in Fig. 10A. Only particles that are at least one particle diameter away from the edges of the image volume were considered for the $Z$ distribution. This is to avoid an underestimation of the average contact number, as there will be many contacts that are missing for the particles identified near the edge. In Fig. 10A, with increasing volume fraction the peak of the distribution shifts to higher values of $Z$. Furthermore, the distribution is almost symmetric for all volume fractions. A similar trend was also reported in a simulation study by Zhang et $a .^{65}$ on soft frictionless emulsion droplets interacting through a repulsive potential. However, introduction of attractive forces between droplets was found to greatly affect the contact number distribution, shifting the peak or $\langle Z\rangle$ to a value below the isostatic value for repulsive frictionless spheres in $3 \mathrm{D}$ $(\langle Z\rangle=6) .{ }^{21}$ The particles with fewer than 4 contacts in the lowest volume fraction samples, for $\varphi<0.726$, represent the rattlers present in the system, which completely vanish at higher compression. The fraction of rattlers at all volume fractions is shown in Table 1 . The value goes to zero at a volume fraction of 0.776 . Note that this value was obtained from the system taking into account the particles that are at least one particle diameter away from the edges of the image volume.

Next, we investigated the scaling of the average contact number $\langle Z\rangle$ with volume fraction $\varphi$, moving away from the jamming point to higher densities. Numerical studies on jamming of frictionless systems found that the contact number obeys a universal power law scaling of the form $Z-Z_{\mathrm{c}}=Z_{0}(\varphi-$ $\left.\varphi_{\mathrm{c}}\right)^{\beta}$, where $\beta=0.5$ for all potentials, dimensions and polydispersities. ${ }^{4,8} Z_{\mathrm{c}}$ is the contact number at the jamming point, or critical volume faction $\varphi_{\mathrm{c}}$. For frictionless particles this is equal to the isostatic value (minimum number of contacts required for mechanical stability), $Z_{\mathrm{c}}=2 D$, where $D=3$ is the dimension of the system. Fig. $10 \mathrm{~B}$ is the plot of $\langle Z\rangle$ with the experimental volume fraction $\left(\varphi=\varphi_{\exp }\right)$ obtained by subtracting the overlap volume from the total volume of the spherical shells. The plot also contains contact numbers measured by excluding the rattlers (marked by *). Except for the lowest volume fraction $\varphi_{\exp }$ $=0.663$ the difference in $\langle Z\rangle$ is rather negligible. We fixed the value of $Z_{\mathrm{c}}=6$ and fitted the data points with the above mentioned power law equation including the rattlers and avoiding the data point at $\varphi=0.663$, since the measured $\langle Z\rangle$ was less than the isostatic value $6(\langle Z\rangle=5.15$ (with rattlers) and $\langle Z\rangle$ $=5.65$ (without rattlers)). The low value of $\langle Z\rangle$ (both with or without rattlers), lower than isostatic value for the packing $\varphi=$ 

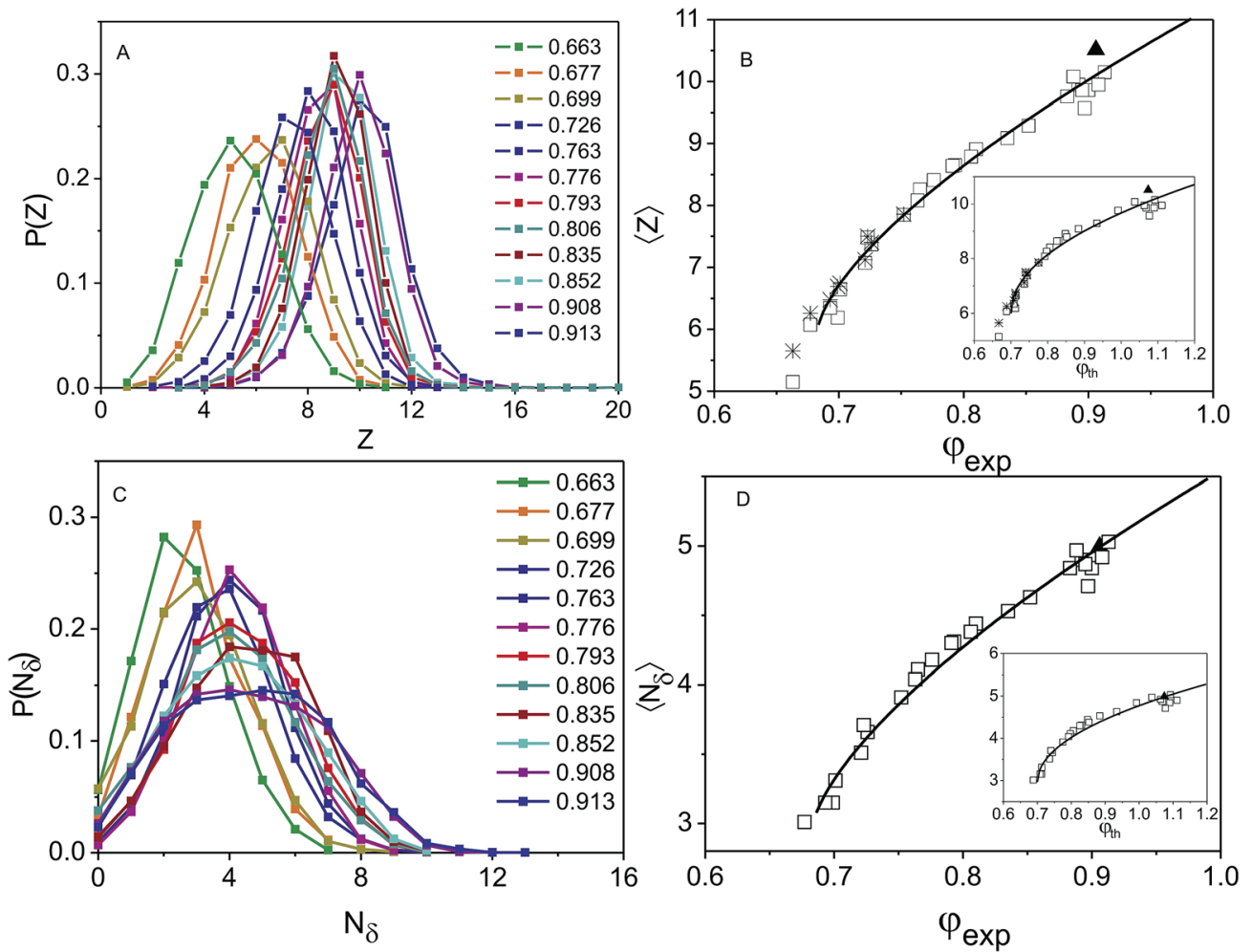

Fig. 10 (A and C) Distribution of contacts per particle, $Z$, and number of dimples per particle, $N_{\delta}$, for a range of experimental packing fractions in the sample of shells of $d / R_{\mathrm{t}}=0.02$. (B) Scaling of the average contact number, $\langle Z\rangle$ with experimental volume fractions $\varphi_{\text {exp. }}$. Solid line is a fit of the form $\langle Z\rangle=6+Z_{0}\left(\varphi-\varphi_{c}\right)^{\beta}$, with an exponent $\beta=0.681$. The data marked in * denote the average value of $Z$ without rattlers. (D) Scaling of the average number of dimples, $\left\langle N_{\delta}\right\rangle$ with experimental volume fraction. The solid lines are a fit to the function: $\left\langle N_{\delta}\right\rangle=6 / x+Z_{0} / x\left(\varphi_{\exp }-\varphi_{c}\right)^{\beta}$ which gives $x=2.024$. Inset plots in (B and $D)$ represent scaling of $\langle Z\rangle$ and $\left\langle N_{\delta}\right\rangle$ with theoretical volume fractions (see text for explanation). The solid triangle in the scaling plots corresponds to the sample of shells of $d / R_{\mathrm{t}}=0.04$.

0.663 , could be a result of error in the determination of particle positions and radii. The error in the measurement of the particle radii means that for particles that are in weak contact we cannot be absolutely certain whether they just touch or not. That gives an error in the contact number. We expect that this gives a slightly low value of $\langle Z\rangle$. Table 2 shows the values of the fit parameters with and without rattlers. The values are nearly the same in both cases. The relatively high value of $\varphi_{\mathrm{c}}=0.683 \pm$ 0.003 compared to random close packing RCP (0.64) results most likely from the uncertainty in the absolute value of the volume fraction obtained from image analysis. Note that an error of just $2 \%$ in the microscope's calibration can already account for this difference in volume fraction. Since, all the volume fraction values have the same systematic deviation, the exponent of the scaling is not expected to be affected by the error in $\varphi$.
The obtained value of the scaling exponent $\beta=0.681 \pm 0.041$ is significantly higher than the value reported from simulations, which is $\beta=0.50$. The value of the prefactor (Table 2) is also higher than the reported simulation value of $Z_{0}=7.7^{4}$ but close to the value found in $3 \mathrm{D}$ compressed polydisperse emulsion $Z_{0}$ $=10.6{ }^{20}$ In the recent article on $2 \mathrm{D}$ foams by Katgert and van Hecke $^{15}$ nearly the same value of $\beta=0.70$ was reported for the scaling of $\langle Z\rangle$ with volume fraction. But these authors also suggested that this results from the discrepancy between the way in which $\varphi$ is calculated in simulations and in experiments. In simulations the volumes of the spheres are fixed and the volume fraction is calculated by counting the number of particles in the periodic box. So when spheres overlap, the overlap volume is counted double, whereas it is only counted once in the experiment and this leads to higher values of $\varphi$. With

Table 2 Values of fit parameters obtained from the power law fit of scaling of $\langle Z\rangle$ with both experimental and theoretical volume fractions

\begin{tabular}{|c|c|c|c|c|}
\hline Fit parameter & $\varphi_{\exp }$ & $\varphi_{\mathrm{th}}$ & $\varphi_{\exp }$ & $\varphi_{\text {th }}$ \\
\hline$\varphi_{\mathrm{c}}$ & $0.683 \pm 0.003$ & $0.700 \pm 0.005$ & $0.681 \pm 0.003$ & $0.695 \pm 0.004$ \\
\hline$\beta$ & $0.681 \pm 0.041$ & $0.490 \pm 0.038$ & $0.653 \pm 0.030$ & $0.485 \pm 0.030$ \\
\hline
\end{tabular}


theoretical $\varphi$ they obtained a scaling exponent $0.50 \pm 0.020,{ }^{15}$ in excellent agreement with simulations.

We similarly converted our experimental $\varphi$ to theoretical value $\varphi_{\text {th }}$ by adding the overlap volume to $\varphi_{\exp }\left(\varphi_{\text {th }}=\varphi_{\text {exp }}+\right.$ $\left.V_{\text {overlap }} / V_{\text {total }}\right)$. With $\varphi_{\text {th }}$ we found a similar power law scaling of $\langle Z\rangle$, shown in the inset plot with exponent $0.490 \pm 0.038$, this time in good agreement with numerical simulations $\mathrm{s}^{4,8}$ and $2 \mathrm{D}$ experiments on frictional particles, ${ }^{9}$ frictionless systems like foams ${ }^{15}$ and emulsions. ${ }^{19,20}$ However, the double counting of overlap volumes is unsatisfactory, and leads to unphysical volume fractions larger than unity. Note that in $2 \mathrm{D}$ frictional disks $^{9}$ and $3 \mathrm{D}$ emulsions ${ }^{20}$ a value of $\beta$ close to 0.50 was found without applying the double counting of overlap volume. In 2D frictional disks ${ }^{9}$ the scaling of $\langle Z\rangle$ was analyzed for a small range of volume fractions $\left(\varphi-\varphi_{c}\right) \sim 0.01$ and within this range $\varphi_{\exp }$ and $\varphi_{\text {th }}$ are nearly equal. Instead, for 3D emulsions $\left(\varphi-\varphi_{\mathrm{c}}\right) \sim$ 0.2 , the same as in our system of shells. However, in emulsions the volume of the droplets are conserved, which is not the case for our shells. We suspect this may be the origin of the higher exponent found in our system of shells. It may be worthwhile to perform simulations on deformable spheres, both volume conserving and non-volume conserving, to resolve this issue.

Since at each contact only one of the shells is dimpled and the other remains spherical, we expect that the average number of dimples, i.e. the buckling number, is half the average number of contacts. And so we expect a similar power law scaling for $\left\langle N_{\delta}\right\rangle$ with volume fraction. The shells with dimples are identified based on the method described in section 3.2.4. Fig. 10D shows a plot of the scaling of $\left\langle N_{\delta}\right\rangle$ with the experimental volume fraction. The line is a power law fit of the form $\left\langle N_{\delta}\right\rangle=6 / x+Z_{0} /$ $x(\varphi-0.683)^{0.681}$, where only $x$ was varied. The best fit gives a value of $x=2.024 \pm 0.008$, as predicted. For the theoretical volume fraction $\varphi_{\text {th }}$ a value of $x=2.026 \pm 0.012$ was found, inset plot in Fig. 10D.

While the average dimple number is simply half the average contact number, there is no simple relation between their distributions. The plots of the distribution of the number of dimples per particle, $N_{\delta}$, at different volume fractions are shown in Fig. 10C. Clearly, for a particle with an average number of contacts, all or none of the contacts or any number in between may result in a dimple. Therefore, the width of the dimple distribution should be wider than half the width of the contact number distribution. This is indeed found to be the case. From the distribution of dimples it is not quantitatively clear yet what fraction of contacts of a particle results in a dimple, and if dimpling one way or the other is a random process. So we determined the probability distribution of the fraction of contacts that leads to a dimple $\left(N_{\delta} / Z\right)$ among all particles with the same contact number in the packing at four different volume fractions (Fig. 11). The symbols indicate the probability of $N_{\delta} / Z$ for the experimental value of $Z$. The lines correspond to a binomial distribution, which in this case is the discrete probability of randomly getting $N_{\delta}$ dimples out of $Z$ contacts given by the form $P\left(N_{\delta}, Z\right)=\left(Z ! /\left(N_{\delta} !\left(Z-N_{\delta}\right) !\right)\right) p^{N_{\delta}}(1-p)^{\left(Z-N_{o}\right)}$, where $p=$ 0.5 , is the probability for a contact of a particle to become a dimple.

For the lowest volume fractions $\varphi=0.726$ and $\varphi=0.763$ the distribution of the fraction of dimpled contacts is in reasonably close agreement with their respective binomial distributions, especially for the value of $Z$ equal to the average value $\langle Z\rangle$ in the packing. At these low volume fractions dimpling appears to be close to random. For higher volume fractions the deviations
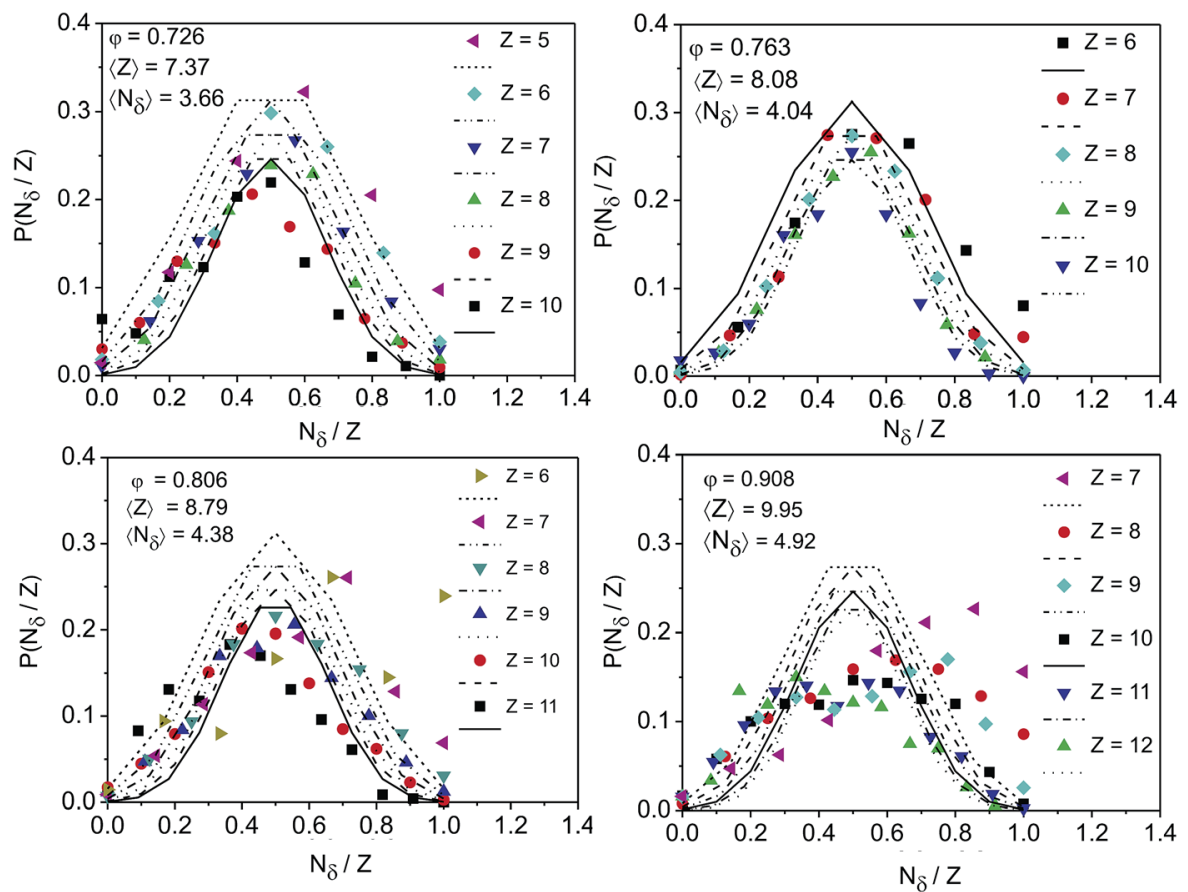

Fig. 11 Distribution of the fraction of contacts that result in a dimple for volume fractions $\varphi=0.726, \varphi=0.763, \varphi=0.806$ and $\varphi=0.908$. Symbols indicate the probability of $N_{\delta} / Z$ of finding $N_{\delta}$ dimples out of $Z$ contacts. The lines represent the binomial distribution for comparison. 
from the binomial distribution become significantly larger, indicating that dimpling becomes non-random. For particles with a number of contacts above or below the average the distribution shows the most deviation from the random probability: the distributions become asymmetric. For $Z<\langle Z\rangle$ the probability is high at large values of $N_{\delta} / Z$ and for $Z>\langle Z\rangle$ the probability is high at small values of $N_{\delta} / Z$. This means that particles with a small number of contacts tend to receive more than their fair share of dimples. Instead, particles having a large number of contacts tend to make a dimple in their neighbors. This behavior can be understood from the fact that the volume enclosed by the shells is not conserved. A particle with many dimples will have a smaller size and can therefore accommodate only a small number of contacting neighbors. Conversely, a particle that has almost no dimples retains its original size and is therefore more likely to be in contact with more than the average number of neighbors.

\section{Conclusions}

We introduced an experimental system consisting of random packings of monodisperse, fluorescently labeled and indexmatched elastic shells under static compression in 3D. Contrary to other jammed systems studied so far these shells do not conserve their volume upon deformation. The shells deform one another by producing dimples in their neighbors. Using confocal microscopy we obtained 3D stacks of images. We developed image processing routines that result in a set of overlapping spheres that closely represents the experimental system. Images were processed in ImageJ software to detect the edges and thereafter analyzing the shape of the particles. This gave the $2 \mathrm{D}$ centers of particles distributed in the 2D slices. The $3 \mathrm{D}$ center of each particle in the full 3D data set was obtained by grouping the $2 \mathrm{D}$ centers and their respective radii and thereafter fitting a circle through the radii of each particle at different positions in the axial direction. A check for pixel biasing revealed the accuracy of the routine in finding the position of the particles with sub-pixel accuracy.

We studied the structure of random packings of these soft shells for a range of experimental volume fractions, above the jamming threshold. The high deformability of the shells enabled us to go far beyond the jamming threshold. The radial distribution function, $g(r)$ for all packings was found to resemble the structure of a liquid where the deformation of the particles broadened the first peak of $g(r)$ and shifted it to radial distances less than the diameter, in contrast to amorphous packings of hard particles. The analysis of the local bond order parameters showed that at all volume fractions the shells were mostly disordered with values in the range of disordered liquids, even the two packings that did not fall in the trend exhibited by other volume fraction samples in $g(r)$. From an analysis of the scaling of the average contact number $Z$ with volume fraction we obtained a higher value of the power law exponent of $\beta=0.68$ than is found in simulations and in some experimental systems. When overlap volumes are added to the volume fractions the predicted value of $\beta=0.50$ is retrieved. We also investigated the scaling of the average number of dimples i.e. the buckling number, $N_{\delta}$. As expected the coefficients of the power law scaling with volume fractions were just half the coefficients in the scaling of average number of contacts $\langle Z\rangle$. This is due to the fact that at each contact only one of the shells is dimpled and the other remained spherical. Further analysis of the randomness of this process of dimpling towards one particle or the other revealed that the process is volume fraction dependent. At higher volume fractions dimpling became gradually non-random, such that particles with a small number of contacts tend to receive more than their share of dimples; on the other hand particles having a large number of contacts tend to make a dimple on their neighbors.

\section{Acknowledgements}

We would like to thank Anjan Gantapara for writing code of grouping of the SEC's and for the help with Mathematica, Johan Stiefelhagen for providing the code for radial distribution function and Bas Kwaadgras for his help in solving programming related issues. Thijs Besseling and Ernest van der Wee are thanked for the local bond order calculations. Nina Elbers is thanked for collaboration on shell synthesis. This work is part of the research program of the Stichting voor Fundamenteel Onderzoek der Materie (FOM), which is financially supported by the Nederlandse organisatie voor Wetenschappelijke Onderzoek (NWO). Gerhard A. Blab is partially supported by the Marie Curie Action (CIG).

\section{References}

1 M. E. Cates, J. P. Wittmer, J. P. Bouchaud and P. Claudin, Phys. Rev. Lett., 1998, 81, 1841-1844.

2 C. H. Liu, S. R. Nagel, D. A. Schecter, S. N. Coppersmith, S. Majumdar, O. Narayan and T. A. Witten, Science, 1995, 269, 513-515.

3 C. S. O'Hern, S. A. Langer, A. J. Liu and S. R. Nagel, Phys. Rev. Lett., 2002, 88, 075507.

4 C. S. O'Hern, L. E. Silbert, A. J. Liu and S. R. Nagel, Phys. Rev. E: Stat., Nonlinear, Soft Matter Phys., 2003, 68, 011306.

5 A. O. N. Siemens and M. Van Hecke, Phys. A, 2010, 389, 42554264.

6 A. J. Liu and S. R. Nagel, Soft Matter, 2010, 6, 2869-2870.

7 M. van Hecke, J. Phys.: Condens. Matter, 2010, 22, 033101.

8 D. J. Durian, Phys. Rev. Lett., 1995, 75, 4780-4783.

9 T. S. Majmudar, M. Sperl, S. Luding and R. P. Behringer, Phys. Rev. Lett., 2007, 98, 058001.

10 T. S. Majmudar and R. P. Behringer, Nature, 2005, 435, 10791082.

11 D. M. Mueth, H. M. Jaeger and S. R. Nagel, Phys. Rev. E: Stat. Phys., Plasmas, Fluids, Relat. Interdiscip. Top., 1998, 57, 3164.

12 D. L. Blair, N. W. Mueggenburg, A. H. Marshall, H. M. Jaeger and S. R. Nagel, Phys. Rev. E: Stat., Nonlinear, Soft Matter Phys., 2001, 63, 041304.

13 J. M. Erikson, N. W. Mueggenburg, H. M. Jaeger and S. R. Nagel, Phys. Rev. E: Stat., Nonlinear, Soft Matter Phys., 2002, 66, 040301. 
14 G. Løvoll, K. J. Måløy and E. G. Flekkøy, Phys. Rev. E: Stat. Phys., Plasmas, Fluids, Relat. Interdiscip. Top., 1999, 60, 5872.

15 G. Katgert and M. van Hecke, Europhys. Lett., 2010, 92, 34002.

16 J. Brujić, S. F. Edwards, I. Hopkinson and H. A. Makse, Phys. A, 2003, 327, 201-212.

17 J. Brujić, S. F. Edwards, D. V. Grinev, I. Hopkinson, D. Brujic and H. A. Makse, Faraday Discuss., 2003, 123, 207-220.

18 J. Zhou, S. Long, Q. Wang and A. D. Dinsmore, Science, 2006, 312, 1631-1633.

19 K. W. Desmond, P. J. Young, D. D. Chen and E. R. Weeks, Soft Matter, 2013, 9, 3424-3436.

20 I. Jorjadze, L.-L. Pontani and J. Brujić, Phys. Rev. Lett., 2013, 110, 048302 .

21 I. Jorjadze, L.-L. Pontani, K. A. Newhall and J. Brujić, Proc. Natl. Acad. Sci. U. S. A., 2011, 108, 4286-4291.

22 K. A. Newhall, I. Jorjadze, E. Vanden-Eijnden and J. Brujić, Soft Matter, 2011, 7, 11518-11525.

23 J. Brujić, C. Song, P. Wang, C. Briscoe, G. Marty and H. A. Makse, Phys. Rev. Lett., 2007, 98, 248001.

24 M. Clusel, E. I. Corwin, A. O. N. Siemens and J. Brujić, Nature, 2009, 460, 611-615.

25 L. D. Landau and E. M. Lifshitz, Theory of Elasticity, Pergamon, New York, 1986.

26 A. V. Pogorelov, Bendings of surfaces and stability of shells, American Mathematical Society, Providence, 1988.

27 F. Caruso, Adv. Mater., 2001, 13, 11-22.

28 S. L. Westcott, S. J. Oldenburg, T. R. Lee and N. J. Halas, Langmuir, 1998, 14, 5396-5401.

29 P. A. L. Fernandes, M. Delcea, A. G. Skirtach, H. Möhwald and A. Fery, Soft Matter, 2010, 6, 1879-1883.

30 C. Y. Gao, E. Donath, H. Möhwald and J. C. Shen, Angew. Chem., Int. Ed., 2002, 41, 3789-3793.

31 J. Zhang, R. J. Coulston, S. T. Jones, J. Geng, O. A. Scherman and C. Abell, Science, 2012, 335, 690-694.

32 C. Li, Z. Y. Li, J. Zhang, K. Wang, Y. H. Gong, G. F. Luo, R. X. Zhuo and X. Z. Zhang, J. Mater. Chem., 2012, 22, 4623-4626.

33 C. I. Zoldesi, C. A. van Walree and A. Imhof, Langmuir, 2006, 22, 4343-4352.

34 C. I. Zoldesi, I. L. Ivanovska, C. Quilliet, G. J. L. Wuite and A. Imhof, Phys. Rev. E: Stat., Nonlinear, Soft Matter Phys., 2008, 78, 051401.

35 M. A. Bevan and S. L. Eichmann, Curr. Opin. Colloid Interface Sci., 2011, 16, 149-157.

36 R. Besseling, L. Isa, E. R. Weeks and W. C. K. Poon, Adv. Colloid Interface Sci., 2009, 146, 1-17.

37 A. van Blaaderen, Prog. Colloid Polym. Sci., 1997, 104, 59-65. 38 V. Prasad, D. Semwogerere and E. R. Weeks, J. Phys.: Condens. Matter, 2007, 19, 113102.

39 M. C. Jenkins and S. U. Egelhaaf, Adv. Colloid Interface Sci., 2008, 136, 65-92.

40 A. van Blaaderen and P. Wiltzius, Science, 1995, 270, 1177.

41 J. C. Crocker and D. G. Grier, J. Colloid Interface Sci., 1996, 179, 298-310.
42 A. Mohraz and M. J. Solomon, Langmuir, 2005, 21, 52985306.

43 A. Kuijk, A. van Blaaderen and A. Imhof, J. Am. Chem. Soc., 2011, 133, 2346-2349.

44 T. H. Besseling, M. Hermes, A. Kuijk, B. de Nijs, T. Deng, M. Dijkstra, A. Imhof and A. van Blaaderen, J. Phys.: Condens. Matter, 2014.

45 S. Sacanna and D. J. Pine, Curr. Opin. Colloid Interface Sci., 2011, 16, 96-105.

46 D. J. Kraft, R. Wittkowski, B. ten Hagen, K. V. Edmond, D. J. Pine and H. Löwen, Phys. Rev. E: Stat., Nonlinear, Soft Matter Phys., 2013, 88, 050301.

47 S. Anthony, L. Zhang and S. Granick, Langmuir, 2006, 22, 5266-5272.

48 S. M. Anthony, M. Kim and S. Granick, J. Chem. Phys., 2008, 129, 244701.

49 N. A. Elbers, J. Jose, A. Imhof and A. van Blaaderen, in preparation.

50 J. Jose, M. Kamp, A. van Blaaderen and A. Imhof, Langmuir, 2014, 30, 2385-2393.

51 ImageJ, http://rsb.info.nih.gov/ij/index.html.

52 T. J. Collins, BioTechniques, 2007, 43, 25-30.

53 M. D. Abràmoff, P. J. Magalhães and S. J. Ram, Biophotonics International, 2004, 11, 36-42.

54 P. J. Steinhardt, D. R. Nelson and M. Ronchetti, Phys. Rev. B: Condens. Matter Mater. Phys., 1983, 28, 784.

55 W. Lechner and C. Dellago, J. Chem. Phys., 2008, 129, 114707.

56 P. R. Ten Wolde, M. J. Ruiz-Montero and D. Frenkel, Phys. Rev. Lett., 1995, 75, 2714.

57 J. Jose, A. van Blaaderen and A. Imhof, to be submitted.

58 S. Hell, G. Reiner, C. Cremer and E. H. K. Stelzer, J. Microsc., 1993, 169, 391-405.

59 T. H. Besseling, J. Jose and A. van Blaaderen, J. Microsc., 2015, 257, 142-150.

60 O. R. Vincent and O. Folorunso, Proceedings of Informing Science and IT Education Conference, 2009, pp. 97-107.

61 E. R. Davies, Machine vision: theory, algorithms, practicalities, Morgan Kaufmann, 2004.

62 J. E. Bresenham, IBM Syst. J., 1965, 4, 25-30.

63 R. J. Hunter, Foundations of colloid science, Oxford University Press, Oxford, 2nd edn, 2001.

64 M. N. van der Linden, D. El Masri, M. Dijkstra and A. van Blaaderen, Soft Matter, 2013, 9, 11618-11633.

65 H. P. Zhang and H. A. Makse, Phys. Rev. E: Stat., Nonlinear, Soft Matter Phys., 2005, 72, 011301.

66 L. E. Silbert, A. J. Liu and S. R. Nagel, Phys. Rev. E: Stat., Nonlinear, Soft Matter Phys., 2006, 73, 041304.

67 G. S. Cargill, J. Appl. Phys., 1970, 41, 2248-2250.

68 T. M. Truskett, S. Torquato, S. Sastry, P. G. Debenedetti and F. H. Stillinger, Phys. Rev. E: Stat. Phys., Plasmas, Fluids, Relat. Interdiscip. Top., 1998, 58, 3083-3088.

69 S. P. Pan, J. Y. Qin, W. M. Wang and T. K. Gu, Phys. Rev. B: Condens. Matter Mater. Phys., 2011, 84, 092201.

70 T. A. Caswell, Z. X. Zhang, M. L. Gardel and S. R. Nagel, Phys. Rev. E: Stat., Nonlinear, Soft Matter Phys., 2013, 87, 012303. 